\title{
Components of reward-driven attentional capture
}

\author{
Li Z. Sha ${ }^{1}$ Yuhong V. Jiang ${ }^{1}$
}

Published online: 16 December 2015

(C) The Psychonomic Society, Inc. 2015

\begin{abstract}
Recent research reported that task-irrelevant colors captured attention if these colors previously served as search targets and received high monetary reward. We showed that both monetary reward and value-independent mechanisms influenced selective attention. Participants searched for two potential target colors among distractor colors in the training phase. Subsequently, they searched for a shape singleton in a testing phase. Experiment 1 found that participants were slower in the testing phase if a distractor of a previous target color was present rather than absent. Such slowing was observed even when no monetary reward was used during training. Experiment 2 associated monetary rewards with the target colors during the training phase. Participants were faster finding the target associated with higher monetary reward. However, reward training did not yield value-dependent attentional capture in the testing phase. Attentional capture by the previous target colors was not significantly greater for the previously high-reward color than the previously low or no-reward color. These findings revealed both the power and limitations of monetary reward on attention. Although monetary reward can increase attentional priority for the high-reward target during training, subsequent attentional capture effects may not be reward-based, but reflect, in part, attentional capture by previous targets.
\end{abstract}

Keywords Selective attention $\cdot$ Visual search $\cdot$ Reward learning $\cdot$ Attentional capture

Li Z. Sha

lixx3632@umn.edu

1 Department of Psychology, University of Minnesota, 75 East River Road, S506 Elliot Hall, Minneapolis, MN 55455, USA

\section{Introduction}

Neurons in many cortical and subcortical regions are sensitive to reward (Shuler \& Bear, 2006; Vickery, Chun, \& Lee, 2011). In humans, secondary reward, such as money, is a powerful motivation for many behaviors. Recent laboratory research has found extensive evidence that monetary reward influences how limited processing resources are prioritized (Anderson, 2013; Chelazzi, Perlato, Santandrea, \& Della Libera, 2013). Anderson, Laurent, and Yantis (2011a) were among the first to show that task-irrelevant stimuli previously associated with high monetary reward captured attention. They asked participants to search for a target color among distractor colors and report the orientation of the line inside the target. The single target on each trial could be either red or green. Unbeknownst to participants, targets in one color frequently yielded a higher monetary reward than targets in the other color. Although participants found both colors equally quickly in the training phase, reward association had long-lasting influences on attention. In a testing phase, participants searched for a unique shape and reported the orientation of the line inside the singleton shape. Color was irrelevant in this phase, but red or green appeared as a distractor color on half of the trials. The presence of red or green slowed down reaction time (RT), relative to when they were absent, demonstrating attentional capture. The high-reward color produced a larger capture effect than the low-reward color (16 vs. $8 \mathrm{~ms}$, although a direct statistical comparison was not reported), suggesting that monetary reward influenced attention.

Value-driven attentional capture launched a large wave of research, with many studies reporting conceptually similar findings to the original results (Anderson, Laurent, \& Yantis, 2011b; Failing \& Theeuwes, 2014; Lee \& Shomstein, 2014; Roper, Vecera, \& Vaidya, 2014; Theeuwes \& Belopolsky, 2012; Wang, Yu, \& Zhou, 2013; see the special issue in Visual 
Cognition, 1-2, 2015). For example, Failing and Theeuwes (2014) showed that after an initial training phase in which one color led to greater monetary reward than the other, the more highly rewarded color later induced greater exogenous cuing. These findings provide compelling evidence that monetary reward is a powerful driver of selective attention (Awh, Belopolsky, \& Theeuwes, 2012; Gottlieb, Hayhoe, Hikosaka, \& Rangel, 2014).

However, these findings were not without controversies. Two issues emerged in the growing literature. First, not all studies supported the initial characterization of attentional capture as value-dependent. Second, whereas some studies found no effects of reward in the training phase, others found that people were faster responding to the more highly rewarded target during training. Here we provide a brief review of these findings before presenting new experimental data to further examine these issues.

Perhaps the most unsettling issue on reward-based attention is whether monetary reward drives attention in a valuedependent or value-independent manner. One independent replication of the value-dependent attentional capture was reported by Jiao and colleagues (Jiao, Du, He, \& Zhang, 2015). These researchers led participants to believe that they were performing the search task simultaneously with another participant, who may receive the same, more, or less reward relative to their own reward. When participants were led to believe that the other individual received the same or less reward, the previously reward-associated colors induced attentional capture in testing, and the magnitude of the capture was greater for the previous high-reward than the previous low-reward color. Value-dependent attentional capture was also observed in Anderson and colleagues' more recent work (Anderson, 2015; Anderson \& Yantis, 2013; Anderson et al., 2011b), and in studies that trained participants to associate reward with a single stimulus (as opposed to a search target; e.g., Mine \& Saiki, 2015). Other studies using primary rewards, such as chocolate odor or electric shock, also have observed value-dependent capture effects by previously reward-associated stimuli (Miranda \& Palmer, 2013; Pool, Brosch, Delplanque, \& Sander, 2014). Contradicting these findings of value-dependency were other studies that showed either no effects of reward training or attentional capture that was value-independent. For example, using Anderson et al.'s (2011a) paradigm, Roper et al. (2014) did not find significant differences among high-reward, low-reward, and baseline conditions in their adult sample. Other studies reported increased capture by previously rewarded stimuli, but the capture effect was not greater for the more highly-rewarded stimulus (Anderson, Laurent, \& Yantis, 2013). In addition, some studies that reported monetary reward driven capture effects did not always report results from the low-reward color, either because this condition was omitted from the design (e.g., Experiment 4 of Sali, Anderson, \& Yantis, 2014) or because no direct statistical comparisons were made between the highand low- reward stimuli (Anderson et al., 2011a). Comparing high- and low-reward stimuli is sometimes made difficult by the small effects of monetary reward. For example, the increased capture effect by a previously high-reward target relative to baseline was only $16 \mathrm{~ms}$ in the original Anderson et al. (2011a) study, leaving little room for the low-reward target to induce a smaller effect.

Why does it matter whether monetary reward modulates attention in a value-dependent or value-independent manner? Although monetary reward could have an "all or none" effect (perhaps because participants treat objectively different rewards as subjectively similar), value-independent results in the original attentional capture paradigm are more difficult to interpret than are value-dependent results. Whereas valuedependent capture directly ties monetary reward with attention, value-independent capture may reflect more general mechanisms unrelated to monetary reward. The high- and low-reward colors were not only rewarded in the training phase, they also happened to be the previous targets. Given the known difficulty of switching search mode and task sets (Leber \& Egeth, 2006; Monsell, 2003), participants may occasionally attend to the previous targets. To rule out the targetdriven capture hypothesis, it is necessary to run comparable experiments that do not involve monetary reward. For example, participants may be trained to search for either red or green targets, without monetary reward, and later be tested in an attentional capture paradigm. Relatively few published studies included this crucial test. The ones that did sometimes reported no attentional capture by former targets (Anderson, Laurent, \& Yantis, 2014; Qi, Zeng, Ding, \& Li, 2013), although some of these studies suffered from low statistical power (Anderson et al., 2011a, Experiment 2, $\mathrm{N}=10$ ). In light of these inconsistencies, it is important to reevaluate how monetary reward modulates selective attention.

A second issue emerging from work on reward-based attention is when reward influenced attention, either during training when differential monetary reward was given, or during testing when monetary reward was removed. Contrary to what one might expect based on previous research on transfer of learning (Perkins \& Salomon, 1992), effects of monetary reward on attention frequently appeared in the testing phase but not the training phase. For example, participants in Anderson et al. (2011a) were equally fast responding to the high and low reward targets during the training phase. This was also the case in several subsequent reports (Anderson \& Yantis, 2013; Gong \& Li, 2014; Sali et al., 2014). Paradoxically, reward learning was expressed subsequently in the testing phase when the previously rewarded colors were taskirrelevant and when monetary reward was no longer used. One explanation for the lack of training effects is that the color search task used in the training phase may have been relatively insensitive. Color search typically has fast RT, leaving little 
room for reward to further reduce RT. The lack of reward effects in the training phase therefore may be attributed to small effect sizes. Consistent with the idea that statistical power may be at play, some studies have found significant effects of monetary reward during the training phase, either because they used a shape discrimination task (Failing \& Theeuwes, 2014 ) or because they associated monetary reward additionally with response (Lee \& Shomstein, 2014). Given that transfer of learning typically depends on having significant training effects to begin with, effects of monetary reward during the training phase merit further investigation.

We present two studies that address the issues raised above. Experiment 1 examined whether, in the absence of monetary reward during training, previous targets can capture attention. This experiment sought evidence for the target-driven capture hypothesis. It leaves open the possibility that monetary reward can influence attention over and above target-driven capture. Experiment 2 tested a large number of participants in a version of the value-driven attentional capture paradigm. Our focus was to (1) examine whether monetary reward influenced performance in the training phase, and (2) test whether attentional capture by previously rewarded targets was value-dependent or value-independent.

\section{Experiment 1}

Using letters as stimuli, previous studies demonstrated that former targets attracted attention in an involuntary manner (Kyllingsbaek, Schneider, \& Bundesen, 2001; Shiffrin \& Schneider, 1977). These findings raised the possibility that the previously reported value-driven capture effects may instead reflect attentional capture by former targets. Experiment 1 examined whether experimental conditions similar to valuedriven attention capture could induce capture effects even when monetary reward was never used. Participants searched for a target that could be in one of two colors, color 1 and color 2 . They received no monetary reward in the training phase. In the testing phase, they performed a singleton-shape search; color was irrelevant to the task. On a subset of the trials, color 1 or color 2 was the color of one of the distractors. We examined whether the presence of previous target colors slowed down search.

\section{Method}

Participants Twenty-four college students (18-35 years, 20 females and 4 males) completed this experiment. The sample size was predetermined to be comparable to or larger than those of previous studies that examined value-driven attentional capture. Participants had normal or corrected-tonormal visual acuity, normal color vision, and were naïve to the purpose of the study.
Equipment Participants were tested individually in a room with normal interior lighting. The experiments were programmed using Psychtoolbox (Brainard, 1997; Pelli, 1997) implemented in MATLAB (www.mathworks.com). Stimuli were presented on a 17" CRT monitor with a resolution of $1024 \times 768$ pixels and a refresh rate of $75 \mathrm{~Hz}$. Viewing distance was unconstrained but was approximately $40 \mathrm{~cm}$.

Materials and Stimuli All stimuli were presented against a black background. Each trial of the training phase presented participants with 6 equidistant outline circles (size: $1.5^{\circ} \times$ $1.5^{\circ}$ ) at an eccentricity of $5^{\circ}$. The colors were chosen from 10 distinctive colors with the constraint that each display had 6 unique colors (Fig. 1A). Two colors were assigned to be the target colors and the others were distractor colors. To control for intrinsic differences in the perceptual salience of different colors, we counterbalanced the color assignments across participants.

In the testing phase, each display contained 6 outline shapes at the same eccentricity as that of the training phase. The display may contain one circle among 5 diamonds (size $1.5^{\circ} \times 1.5^{\circ}$ ) or one diamond among 5 circles. All six shapes had different colors drawn from the same general set as the training phase, with the constraint that the unique shape (target) could not have the same color as the target colors used in the training phase.

Inside each outline circle or diamond was a white vertical or horizontal line (length: $1^{\circ}$ ). Its orientation was randomly chosen (Fig. 1B).

Procedure Participants completed the training and testing phases on two consecutive days. In the training phase, participants were shown an array of 6 colored circles and were asked to find the target circle defined by its color. They then pressed a button to report the orientation of the line inside the target circle. The target could be one of two prespecified colors. Only one target appeared on each trial and it was equally likely to be either of the two prespecified colors. There were 768 training trials. One day later, participants returned for the testing phase for a total of 300 trials. This time they searched for an odd shape (either the unique circle among five diamonds or the other way around, presented in a random order) and pressed a button to report the orientation of the enclosed line.

Each visual search trial started with a white fixation point $\left(0.5^{\circ} \times 0.5^{\circ}\right)$ with a random duration between 400 and $600 \mathrm{~ms}$. The search display was then shown until participants pressed a button corresponding to the orientation of the target's line. To discourage errors, the computer voice spoke the sentence "That was wrong. Please try to be accurate" after each incorrect response. Correct trials were followed by three pure tones (800, 1300, and $2000 \mathrm{~Hz}$, each for $100 \mathrm{~ms}$ ) or the text "too slow," depending on whether RT was faster than the cutoff of 


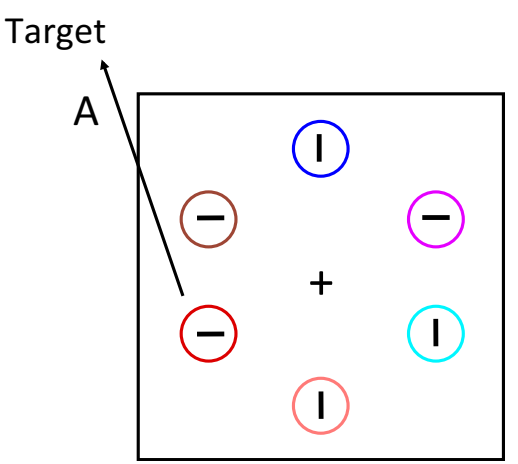

Fig. 1 Schematic illustrations of the search displays. A Training phase. The target was defined by its color (e.g., red or green). B Testing phase. The target was the shape singleton. The previous target colors may be

$1,000 \mathrm{~ms}$ in the training phase or $1,500 \mathrm{~ms}$ in the testing phase. These cutoff times were chosen because pilot testing using more stringent cutoffs led to error rates higher than $10 \%$. The next trial commenced after 1,000 ms. Participants were given a break every 48 trials in the training phase and every 60 trials in the testing phase.

Design Unlike value-driven capture studies, participants in Experiment 1 received no monetary reward in the training phase. Color 1 and color 2 were therefore simply target colors. In the testing phase, participants searched for an odd shape and were asked to ignore item colors. The target was a circle among diamonds on half of the trials, and a diamond among circles on the other half, in a randomly mixed order. Its color was randomly selected except that it could not be the two target colors used in the training phase. The previous target colors were present on $40 \%$ of the trials as a distractor $(20 \%$ color $1,20 \%$ color 2) and absent on the other $60 \%$ of the trials (baseline). Trial types were randomly intermixed in presentation order. No monetary reward was involved in the testing phase either.

\section{Results}

\section{Training phase}

Accuracy in the training phase was $94.9 \%$. Figure 2 (left) displays RT data for correct trials, excluding trials in which RT was longer than $10 \mathrm{~s}(0.12 \%$ of the data were removed as outliers). RT for color 1 and color 2 was equivalent, $F<1$. RT improved as training progressed, yielding a significant main effect of block, $F(15,345)=2.15, p<0.01, \eta_{p}{ }^{2}=0.09$. Color and block did not interact, $F<1$.

\section{Testing phase}

We next examined whether previous target colors captured attention in the testing phase. Accuracy in the testing phase

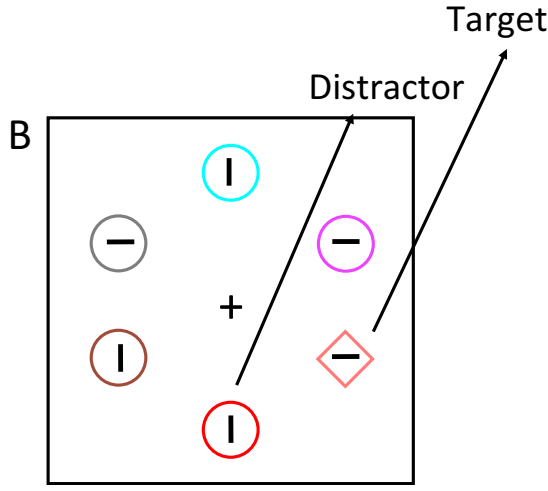

absent (baseline) or present (as illustrated). Participants reported the orientation of the line inside the target in both phases

was comparable for trials in which a distractor in color 1 or color 2 was present $(91 \%)$ and trials in which they were absent $(91 \%), t<1$. Figure 2 (right) shows RT for correct trials. Even though monetary reward was not involved during training, the target colors used in the training phase slowed down search in the testing phase, relative to when they were absent, $t(23)=2.10, p<0.05$, Cohen's $d=0.88$. In a further analysis, we separated data from the first and the second half of the testing phase. Distractor color condition did not interact with testing half, $F<1$, indicating a consistent pattern of results in both halves of testing.

\section{Discussion}

Experiment 1 showed that following a color search task, participants were susceptible to attentional capture by the previous target colors. In the testing phase when they searched for a singleton shape, the presence of the previous target colors in one of the distractors slowed down search. Consistent with previous studies using letter stimuli, this finding indicates that previous targets can capture attention (Kyllingsbæk, Schneider, \& Bundesen, 2001; Shiffrin \& Schneider, 1977). It fits with the widely accepted ideas that switching one's attentional set is challenging (Leber \& Egeth, 2006; Monsell, 2003) and that attentional capture is influenced by the attentional control setting (Folk, Remington, \& Johnston, 1992).

Experiment 1 demonstrates that experimental conditions similar to those used in value-driven capture studies also are conducive to target-driven capture. These findings reinforced the idea that former targets attracted attention (Kyllingsbaek et al., 2001; Shiffrin \& Schneider, 1977). They may seem inconsistent with some previous reports, which did not find attentional capture by previously unrewarded targets (Anderson et al., 2011a; Anderson, et al., 2014; Qi, et al., 2013; Sali et al., 2014). One explanation for the inconsistency is that the large 

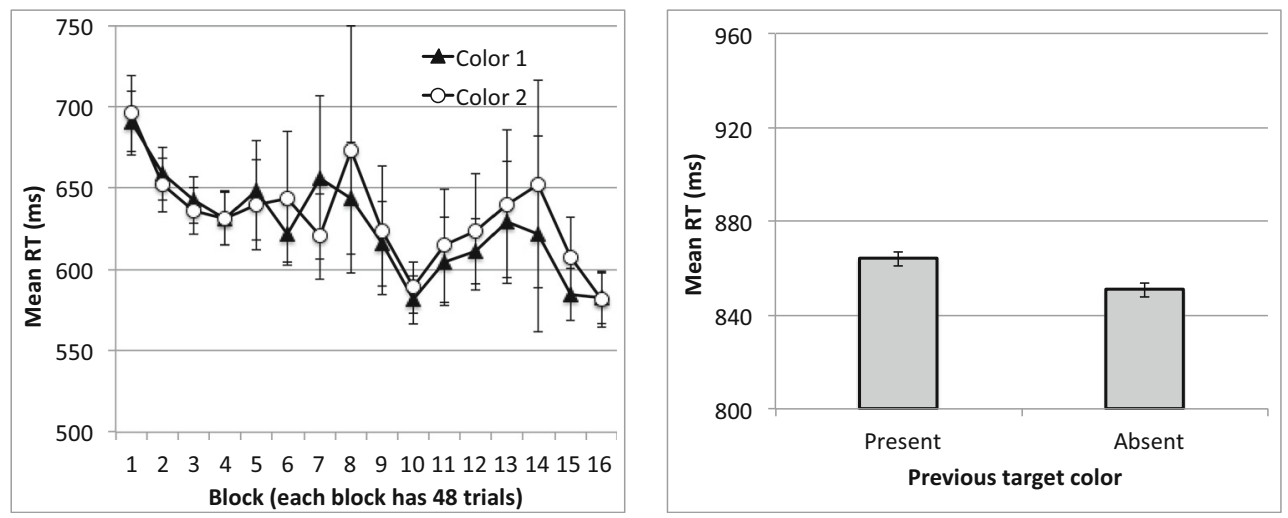

Fig. 2 Results from Experiment 1. Left: Training phase. Right: Testing phase. Error bars show \pm 1 within-subject S.E. of the mean

sample size used in our experiment may have given us sufficient statistical power to detect target-driven capture. Our results, however, were consistent with one previous study on value-driven attentional capture (Wang et al. 2013). In this study, Wang et al. included a control experiment in which participants searched for two potential targets, without receiving any monetary reward. In a subsequent testing session, participants were slower finding a singleton target when the display included a former search target, than when the display included a novel distractor. Our study differed from Wang et al. in our choice of the baseline condition. Whereas Wang et al. included a novel stimulus as their baseline, our study omitted the former targets without adding a novel item. Given that novel stimuli could accentuate capture (Folk \& Remington, 2015; Horstmann \& Ansorge, 2006; Neo \& Chua, 2006), Wang et al. may have underestimated the target-induced capture effect. What is in common, however, is that both studies revealed attentional capture by former targets associated with no monetary reward.

Results of Experiment 1 constrain the kind of inferences and conclusions one can draw when evaluating capture effects by a previously rewarded target. These effects could have two components: target-driven capture and value-driven capture. To isolate the value-driven component it is not enough to compare reward-target absent trials with reward-target present trials. Rather, one would need to examine whether capture effects are stronger for a high reward target than a lowreward or no-reward target.

\section{Experiment 2}

Having demonstrated that previous targets could induce attentional capture (Kyllingsbaek et al., 2001; Shiffrin \& Schneider, 1977; Wang et al. 2013), we tested whether attentional capture was stronger for a target that received greater monetary reward than one that received less or no monetary reward. In the training phase participants received monetary reward upon finding the target. Experiment 2A manipulated monetary reward probabilistically. One target color received a high reward $80 \%$ of the time and a low reward $20 \%$ of the time, whereas the other target color received a high reward $20 \%$ of the time and a low reward $80 \%$ of the time. This version contrasted high-reward with low-reward. Experiment 2B manipulated reward in an all-or-none fashion. One target color received a high reward $100 \%$ of the time and the other target color received no reward $100 \%$ of the time. This version contrasted high reward with no reward. ${ }^{1}$ In both versions, the two colors were target colors. Target-driven capture effects should be comparable between the two colors. Any difference between them would correspond to value-dependent capture effects.

In addition to examining attentional capture by previously rewarded targets in the testing phase, Experiment 2 aimed to delineate reward learning during the training phase. The large sample size (48 across Experiments 2A and 2B) increased our chance to detect an effect of monetary reward during training. Charting out the learning curve, as opposed to reporting just the mean RT across the entire training phase, also enabled us to examine the consistency of reward learning across different blocks of training.

Experiments 2A and 2B differed in minor aspects; these variations were designed to ensure that our results were not specific to parameters used in either experiment. Training was long in Experiment 2A (768 trials, similar to Anderson et al., 2011a's Experiment 1) but shorter in Experiment 2B (384 trials, similar to Anderson et al., 2011a's Experiment 3). Although longer training may be expected to produce greater effects of learning, one anonymous reader noted that longer training could accentuate target-driven capture effects.

In addition, testing was administered either after a 1-day delay (Experiment 2A) or immediately (Experiment 2B). The longer delay may facilitate consolidation of reward learning, yet the shorter delay may reduce forgetting. We thus tested

\footnotetext{
${ }^{1}$ We thank Jan Theeuwes for suggesting this manipulation.
} 
both long and short delays. Finally, feedback for incorrect trials took the format of either a spoken message ("That was wrong. Please try to be accurate" in Experiment 2A) or a pure tone (Experiment 2B). The spoken messages may be more effective in discouraging errors, reducing speed-accuracy tradeoff. However, they were not typically used in previous studies on value-driven attentional capture. Therefore, we used pure tones for accuracy feedback in Experiment 2B.

\section{Method}

Participants Forty-eight participants completed this experiment: 24 in Experiment 2A (14 females and 10 males) and 24 in Experiment 2B ( 15 females and 9 males). Participants were aged 18-35 years, had normal or corrected-to-normal visual acuity, normal color vision, and were naïve to the purpose of the study.

Stimuli and materials We used the same stimuli as those of Experiment 1.

Procedure Trial sequences were similar to those of Experiment 1 in the training phase, except that participants received monetary reward after each trial. Each correct response that met the RT cutoff of $1,000 \mathrm{~ms}$ led to a display of reward earned. In Experiment 2A, the reward was either high " +10 " (font size 50) or low " +1 " (font size 20), displayed at the center of the screen for $500 \mathrm{~ms}$. In Experiment 2B, the reward was either high " +10 " (font size 50 ) or zero " +0 " (font size 20). Cumulative reward was displayed at $5^{\circ}$ below the trial reward.

In addition to the presentation of just high or no reward, Experiment 2B differed from Experiment 2A in that we modified the computer's feedback. In Experiment 2A, an incorrect response was followed by the computer speaking the sentence "That was wrong. Please try to be accurate." A correct response made within the RT cutoff was followed by three short tones $(800,1300$, and $2000 \mathrm{~Hz}$, each for $100 \mathrm{~ms})$. In Experiment $2 \mathrm{~B}$, an incorrect response was followed by a low tone $(400 \mathrm{~Hz}, 200 \mathrm{~ms})$ and a correct response made within the RT cutoff was followed by a high tone $(1000 \mathrm{~Hz}, 200 \mathrm{~ms})$.

Participants were told that they would receive monetary reward indicated by the number, but only if they made a correct response within the cutoff time. Trial sequences used in the testing phase were the same as those of Experiment 1.

Design Experiments 2A and 2B differed slightly in how monetary reward was given in the training phase. In Experiment $2 \mathrm{~A}$, reward was given probabilistically to the two target colors. The high-reward target color was followed by " +10 " $80 \%$ of the time and " +1 " $20 \%$ of the time. The low-reward target color was followed by " +1 " $80 \%$ of the time and " +10 " $20 \%$ of the time. Similar to Experiment 1, the high- and low- reward colors appeared equally often. Reward accumulated to a point total and was converted into cash payment at the completion of the experiment using a sliding scale ( $\$ 2$ for 0-3999 points, $\$ 3$ for $4000-4499$ points, and $\$ 4$ for 4500 points and above). Participants also were compensated for their time ( $\$ 10 /$ hour or extra course credit). The amount of cash reward used in our study was comparable to that of previously published results (Failing \& Theeuwes, 2014; Stankevich \& Geng, 2014). Providing points, then converting them to cash, had been used previously to induce reward-based attention (Wang et al., 2013; Wentura, Müller, \& Rothermund, 2013). We did not tell participants that one color would yield more monetary reward than the other. Like Experiment 1, the testing phase occurred 1 day later.

In Experiment 2B, reward was given exclusively to one target color. The high-reward target color was followed by "+10" $100 \%$ of the time, and the no-reward target color was followed by " +0 " $100 \%$ of the time. Training lasted 384 trials ( 8 blocks) rather than 768 trials. To reduce forgetting, we administered the testing phase immediately after the training phase rather than 1-day later. Other aspects were the same as in Experiment 2A.

Recognition At the completion of the experiment, participants were asked to estimate the percentage of trials that a highreward followed each of the two target colors. A higher estimation for the high-reward color was considered correct.

\section{Results}

\section{Training phase}

Mean accuracy was $95.1 \%$ for both target colors in Experiment $2 \mathrm{~A}, 93.3 \%$ for the high-reward color, and $92.6 \%$ for the no-reward color in Experiment 2B. Reward did not influence accuracy, $t<1$ in both Experiments 2A and 2B. Figure 3 shows RT data for correct trials (all trials had RT faster than the 10 -s cutoff time).

Monetary reward had a clear effect on search RT in the training phase. With 48 participants across Experiments $2 \mathrm{~A}$ and $2 \mathrm{~B}$, we observed a significant main effect of reward in Blocks 1-8, $F(1,46)=7.36, p<0.01, \eta_{p}{ }^{2}=0.14$. The effect was consistent across the two experiments and did not interact with experimental version, $F<1$. RT became faster in later blocks, yielding a significant main effect of block, $F(7,322)=$ $36.55, p<0.001, \eta_{p}{ }^{2}=0.44$. Block did not interact with reward or experimental version, largest $F(7,322)=1.69, p>$ 0.10 .

Extending the training further in Experiment 2A continued to yield consistent RT differences between the high- and lowreward colors. In Blocks 9-16 RT was significantly faster for the high-reward color than the low-reward color, $F(1,23)=$ 4.54, $p<0.05, \eta_{p}{ }^{2}=0.17$, and this effect did not interact with 

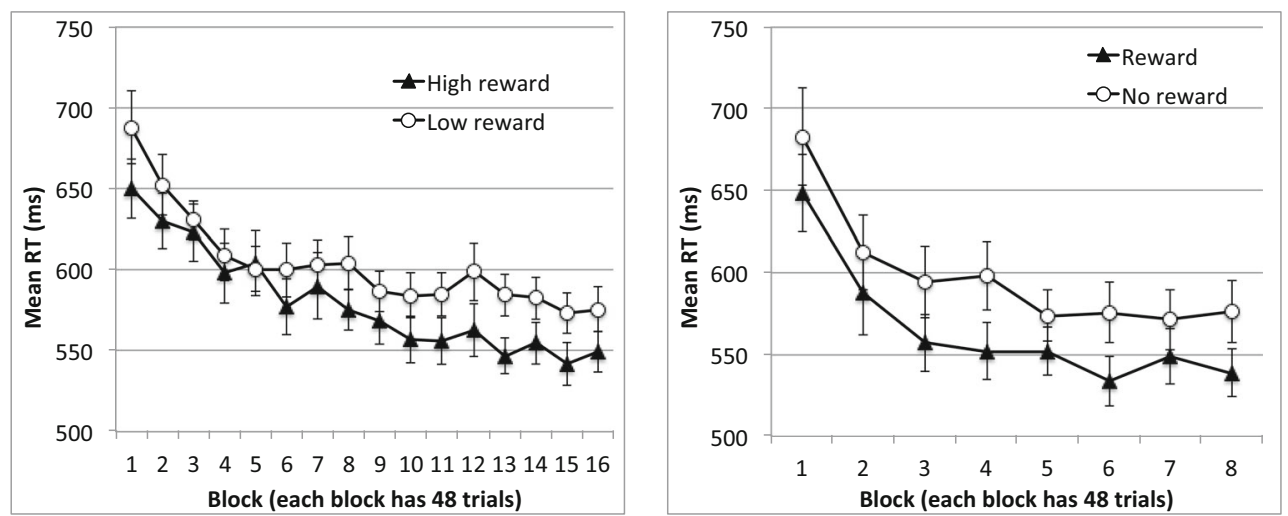

Fig. 3 Results from the training phase of Experiment 2. Left: Data from Experiment 2A; Right: Data from Experiment 2B. Error bars show \pm 1 withinsubject S.E. of the mean

block, $F<1$ for the interaction between reward condition and block.

Thus, consistent with some previous studies, monetary reward influenced search behavior during the training phase (Le Pelley, Pearson, Griffiths, \& Beesley, 2015; Failing \& Theeuwes, 2014; Lee \& Shomstein, 2014). Participants were faster responding to the more highly rewarded color. Reward facilitation emerged relatively early, reaching statistical significance in the first block, $t(47)=2.06, p<0.05$, Cohen's $d=$ 0.60 .

The early onset of reward effects in the training phase could not be attributed to intrinsic differences between the highreward and the low/no reward colors. The counterbalancing method for colors used in Experiment 2 was the same as in Experiment 1, which revealed comparable RTs for the two target colors. This suggests that the faster RT for the highreward color was driven by monetary reward rather than by extraneous factors.

One mechanism that can produce faster RT for the highreward target is repetition priming. Previous studies showed that RT was faster when the target color repeated, rather than changed, on consecutive trials (Kristjánsson \& Campana, 2010; Maljkovic \& Nakayama, 1994). In addition, some studies found that priming was strengthened following a high reward (Della Libera \& Chelazzi, 2006, 2009; Hickey, Chelazzi, \& Theeuwes, 2014; Kristjánsson, Sigurjónsdóttir, \& Driver, 2010). Reward-modulation of priming, however, was unlike an explanation for our study. Across all participants (Blocks 18), RT was facilitated when the current trial's target color was the same, rather than different, as the preceding trial's target color, $F(1,46)=14.33, p<0.001, \eta_{p}{ }^{2}=0.24$ for the main effect of repetition. However, repetition priming was not greater following a high-reward color (mean $16 \mathrm{~ms}$ ) than following a low/no-reward color (mean $23 \mathrm{~ms}$ ), resulting in a lack of interaction between reward and repetition, $F(1,46)=$ $1.43, p>0.23$. This finding suggests that monetary reward may have directly increased the attentional priority for the high-reward target, rather than indirectly by increasing repetition priming.

\section{Testing phase}

The nature of the distractor color in the testing phase did not significantly influence search accuracy (Table 1$), F(2,94)=$ $1.22, p>0.30$.

The use of two targets that received different reward enabled us to separate target-driven capture from value-driven capture effects (Fig. 4). ANOVA on distractor color condition (high-reward, low/no reward, or absent) and experimental version $(2 \mathrm{~A}$ or $2 \mathrm{~B})$ revealed a significant main effect of distractor color condition, $F(2,92)=6.60, p<0.01, \eta_{p}{ }^{2}=0.13$. This effect was comparable between the two versions, with no interaction between color condition and experimental version, $F<1$, suggesting that the two experiments produced similar results. Dividing the testing phase into the first and second half revealed no interaction between half and distractor color condition, $F<1$ for both Experiments $2 \mathrm{a}$ and $2 \mathrm{~b}$.

To understand the significant effects of distractor color, we performed two planned contrasts. Experiment 2A used highand low-reward targets, whereas Experiment 2B used highand no-reward targets. To capture possible differences between the two versions we conducted the analysis separately for Experiments 2A and 2B. Target-driven capture was evaluated by comparing the previous target present condition with the previous target absent condition. We found that RT was

Table 1 Mean accuracy in the testing phase of Experiment 2

\begin{tabular}{llll}
\hline Experiment & $\begin{array}{l}\text { High-reward } \\
\text { color }\end{array}$ & Low/no- reward color & Absent \\
\hline $2 \mathrm{~A}$ & $92.5 \%(1.0 \%)$ & $93.2 \%(1.1 \%)$ & $93.2 \%(0.7 \%)$ \\
$2 \mathrm{~B}$ & $86.9 \%(1.6 \%)$ & $87.8 \%(1.3 \%)$ & $88.4 \%(1.1 \%)$ \\
\hline
\end{tabular}

Standard error of the mean is shown in the parenthesis 

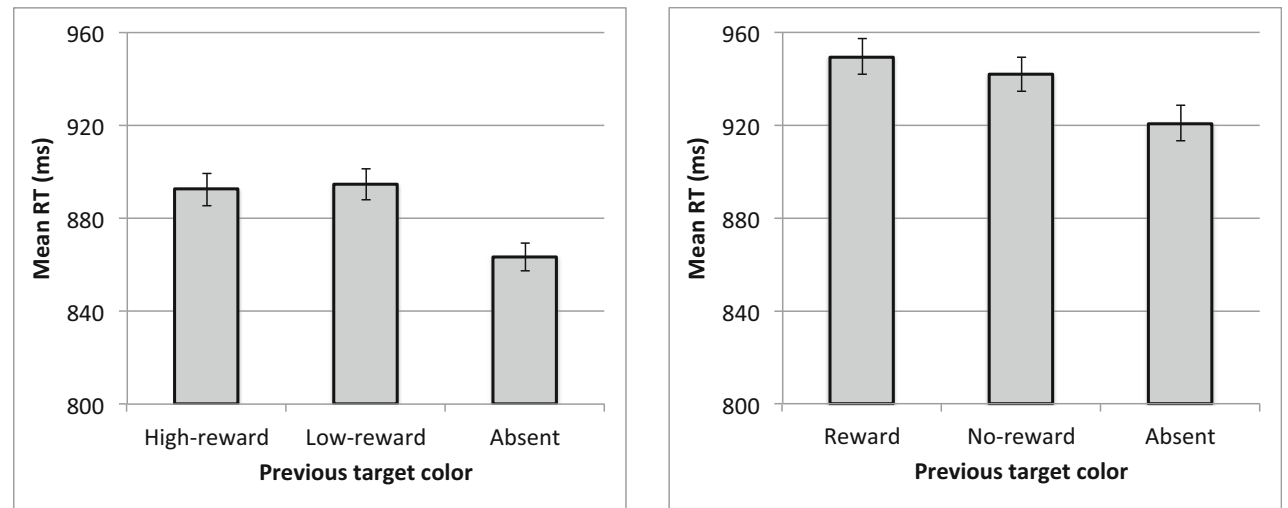

Fig. 4 Results from the testing phase of Experiment 2. Left: Data from Experiment 2A; Right: Data from Experiment 2B. Error bars show \pm 1 withinsubject S.E. of the mean

significantly slower when the previous target color was present rather than absent, in both Experiment $2 \mathrm{~A}(t(23)=3.22$, $p<0.01$, Cohen's $\mathrm{d}=1.34)$ and Experiment $2 \mathrm{~B}(t(23)=2.12$, $p<0.05$, Cohen's $\mathrm{d}=0.88$ ). Value-dependent capture effect was evaluated by comparing the previous high-reward condition with the previous low/no-reward condition. The RT difference of $2 \mathrm{~ms}$ did not reach significance in Experiment 2A $(t(23)=-0.16, p>0.85)$, nor did the RT difference of $7.5 \mathrm{~ms}$ in Experiment $2 \mathrm{~B}$ reach significance $(t(23)=0.58, p>0.55)$. Similar results were found when data were pooled across all 48 participants. These analyses suggested that attentional capture observed in Experiment 2 reflected primarily targetinduced capture and that the capture effect was largely value-independent.

The data presented so far suggested that high- and low- (or no-) reward targets induced comparable attentional capture in the testing phase, suggesting that the capture effect was valueindependent. However, did the use of monetary reward enhance the overall capture effect, relative to Experiment 1 in which no money was used? To address this question, we performed a cross-experiment comparison for the testing phase data between Experiment $1(\mathrm{~N}=24)$ and Experiment $2(\mathrm{~N}=$ 48). We entered color condition (previous target present vs. previous target absent) as a within-subject factor and experiment as a between-subject factor in an ANOVA. This analysis revealed a significant main effect of color condition, with slower RT when the previous target color was present rather than absent, $F(1,70)=9.66, p<0.01, \eta_{p}{ }^{2}=0.12$. The main effect of experiment was not significant, $F(1,70)=1.47, p>$ 0.20 , neither did experiment interact with color condition, $F<$ 1. Restricting the cross-experiment comparison to just Experiment 1 and Experiment $2 \mathrm{~A}$ revealed the same results: experiment did not interact with color condition, $F(1,46)=1.45, p$ $>0.23$. Similarly, restricting the comparison between Experiment 1 and just the high-reward condition of Experiment $2 \mathrm{~B}$ and high/low-reward conditions of Experiments $2 \mathrm{~A}$ revealed no interaction between experiment and color condition, $F(1$, $70)=1.40, p>0.24$. Thus, the use of monetary reward did not induce statistically greater attentional capture in Experiment 2 relative to Experiment 1.

\section{Role of explicit awareness}

Twelve of the 24 participants in Experiment 2A, and 14 of the 24 participants in Experiment $2 \mathrm{~B}$ assigned a higher reward value to the high-reward color than the low/no-reward color. These were the "aware" participants. The other participants assigned either equal or a lower value to the high-reward color. These were the "unaware" participants. To examine whether explicit awareness influenced search data, we performed an additional analysis that included recognition accuracy as a between-subject factor. In neither the training nor the testing phase did recognition accuracy interact with effects of reward, all $p$-values $>0.10$. This finding suggests that explicit knowledge was not a critical factor in our study.

\section{Discussion}

Using a large sample of 48 participants, Experiment 2 showed that monetary reward influenced search behavior during the training phase. RT was faster for the target color associated with greater monetary reward than for the other target color. This effect emerged early and remained stable across 16 blocks of training. This finding supported the idea that monetary reward can influence attentional priority in visual search (Anderson, 2013; Chelazzi et al., 2013; Bucker et al., 2015; Le Pelley et al., 2015; Theeuwes \& Belopolsky, 2012). In the dual-target search task used here, monetary reward may have influenced the search template by giving higher weights to the high-reward target (Hout, Walenchok, Goldinger, \& Wolfe, 2015; Menneer, Cave, \& Donnelly, 2009; Vickery, King, \& Jiang, 2005).

Although monetary reward influenced search performance in the training phase, in our study it did not have durable effects in a subsequent transfer task. When performing shape singleton search in the testing phase, participants 
demonstrated attentional capture by previous target colors. The degree of capture, however, was not significantly greater for the previously high-reward target color than the previously low/no-reward target color. These results held across variations in the length of training, delay between training and testing, and format of accuracy feedback. These findings constrained interpretations of value-driven attentional capture. Although monetary reward can influence attention (as evidenced in the training phase), its impact on subsequent attentional capture may be weaker than previously thought. ${ }^{2}$

In our study, points were converted into cash payment at the end of the experiment. The amount of cash reward used here was comparable to those used in previously published work (Failing \& Theeuwes, 2014; Stankevich \& Geng, 2014). In addition, some studies showed that points could modulate attention (Shen \& Chun, 2011; Wang et al., 2013; Wentura et al., 2013). The significant effect of monetary reward on training phase performance further showed that the reward manipulation was effective in affecting behavior. Future studies should examine whether value-driven attention capture is stronger when monetary reward is substantially greater than what was used in our study.

Our study revealed attentional capture in the testing phase, both when no money was involved in the training phase (Experiment 1) and when money was used (Experiment 2). In addition, capture effects were statistically equivalent between the two experiments. In a previous study, Wang et al. (2013) also observed attentional capture both with and without monetary reward, though the former was statistically stronger. As noted earlier, one difference between these two studies was the definition of "baseline" for evaluating attentional capture. Whereas Wang et al. included a novel stimulus as the baseline, we used target-absent as the baseline. Our design therefore allowed us to assess capture by former targets, independent of potential capture by novel stimuli (Folk \& Remington, 2015; Horstmann \& Ansorge, 2006; Neo \& Chua, 2006). It is important to note that our argument about value-independent capture rested primarily on the withinexperiment comparison of high-reward and low-reward colors. A between-experiment comparison may be open to generic, value-independent effects of reward, such as increased motivation or arousal for participants tested in the context of monetary reward. In the general discussion we will

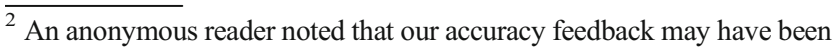
too strong, akin to aversive outcomes (Schmidt, Belopolsky, \& Theeuwes, 2015a, 2015b) or arousing sounds (Miranda \& Palmer, 2013) known to influence attention. We believe that this is unlikely. The sounds used as accuracy feedback were pure tones. Unlike whizzing or hammering sounds used in Miranda \& Palmer (2013), the pure tones had no prior value. The computer's spoken message ("That was wrong; please try to be accurate") was given by the computer's monotone. It is unlikely to induce the kind of aversive response associated with electric shock (Schmidt et al., 2015a, 2015b). Experiment 2b also simplified the feedback to just a simple tone.
}

discuss other factors that may modulate value-driven attentional capture.

\section{General Discussion}

This study examined the role of monetary reward in modulating selective attention to visual features (color). Experiment 1 showed that previous target colors interfered with subsequent shape search, even though monetary reward was not used in any part of the experiment. Experiment 2 showed that associating differential monetary reward to two possible target colors induced reward learning in the training phase. RT was faster for the more highly rewarded color. In a subsequent shape search task, the presence of these colors as distractors slowed down RT. Consistent with a subset of previous findings, the degree of attentional capture by previous target colors was value independent-it was comparable between the previously high-reward and low-reward or no-reward colors (see also Anderson et al., 2013). The valueindependent capture effect implicated general mechanisms, such as the capture by previous target colors, as contributing factors to value-driven attentional capture.

Our study addressed previously conflicting findings regarding value-driven attentional capture. First, we showed that previous target colors could capture attention even though monetary reward was not used. Studies that previously did not assess the target-driven capture effect therefore are open to alternative interpretations. Second, our experimental design enabled us to partition value-dependent and target-driven capture effect. The testing phase data showed a significant targetdriven capture effect, but not a significant value-dependent capture effect. Third, although we failed to find significant value-dependent effects in the testing phase, the training phase data provided clear evidence that monetary reward could influence attention. The more highly rewarded target color produced faster search RT, and this effect was unrelated to explicit knowledge about the reward association (see also Le Pelley et al., 2015).

Our study supports a more nuanced view about valuedriven attention than is sometimes suggested in the literature. On the one hand, data from the training phase of our study reinforces a growing literature showing that monetary reward can influence attention (Anderson et al., 2011a; Anderson \& Yantis, 2013; Chelazzi et al., 2013; Failing \& Theeuwes, 2014; Le Pelley et al., 2015; Theeuwes \& Belopolsky, 2012). The two-target search task used here is a form of "hybrid" memory and perceptual search, with the two potential targets held in memory when perceptually searching through an array of items (Wolfe, 2012). Previous studies using this paradigm showed that search was faster for the more prevalent target than for the other target (Godwin, Menneer, Riggs, Cave, \& Donnelly, 2015; Hout et al., 2015). We 
extended these findings by showing that higher monetary reward could produce similar results. These data indicated that monetary reward influenced the weight assigned to the two potential targets in a hybrid search task.

On the other hand, although monetary reward affected search performance in the training phase, our study suggested that its impact was negligible in the transfer phase. Target colors used in the training phase induced attentional capture in a shape search task. However, the degree of attentional capture was not greater for the high-reward color than for a color that was less or not rewarded.

Our results do not rule out the possibility that other forms of reward, such as primary reward or social reward, may exert stronger effects on attention. In addition, they also leave open the possibility that value-independent effects may involve reward processing. Some researchers have linked target detection to reinforcement learning (Seitz \& Watanabe, 2005; Swallow \& Jiang, 2013). One may argue that the previous targets captured attention not because they were previously attended, but because they were previously associated with successful task completion, especially when strong accuracy feedback was used. However, such effects clearly go beyond monetary reward and should not be taken as evidence for value-driven attention. In addition, labeling all effects as reward-related risks obscures important differences among potentially different sources of reinforcement learning, reducing rather than increasing the explanatory power of reward processing.

Although we did not find evidence for value-dependent attentional allocation in the testing phase, it remains possible that value-dependency can be found in other experimental paradigms or when using stronger reward manipulation. Le Pelley and colleagues showed that a distractor stimulus associated with high monetary reward induced attentional capture (Le Pelley et al., 2015). This effect cannot be attributed to target-driven capture. Similar to our study, however, the effect was observed during training rather than in a transfer test. Other studies in the literature reported value-driven capture effects. Some of these studies used different measures (e.g., first saccadic eye movement in Theeuwes \& Belopolsky, 2012) or different assessments of attention (e.g., exogenous cuing in Failing \& Theeuwes, 2014) than what was used in the current study. The diversity of findings in the literature raises the possibility that training using monetary reward can yield transferrable effects in some, but not all, measures of attention. An important future research direction is to examine when such transferrable effects are robustly found.

One factor that may mediate attentional capture by previously rewarding stimuli is the nature of reward training. Compelling evidence for greater capture by previously high-reward stimuli had been observed when no search was involved in the training phase. For example, Pool et al. (2014) associated one shape with chocolate odor (CS+) and another shape with just air (CS-). Subsequently, CS+ shape induced attentional shifting. In another study, Wentura et al. (2013) presented colors one at a time, and associated different colors with different amounts of monetary reward. Subsequently, they found that colors associated with higher reward induced greater capture. Finally, participants in a study by Mine and Saiki (2015) responded to a centrally presented target, whose color was associated with various levels of monetary reward. This study, too, evidenced value-dependent capture in a testing phase. What was in common among these studies was that participants did not perform any visual search during reward training. Because the reward-associated stimuli cannot be considered previous targets (no search was performed on them), they are unlikely to produce target-induced capture. In addition, effects of monetary reward on attention may be stronger when reward learning was the primary process in the training phase, as opposed to a process secondary to visual search. Future studies should test the role of different training procedures in inducing value-driven attentional capture.

\section{Conclusions}

We have provided evidence that attentional capture by previously rewarded search targets may be primarily target-driven. Capture effects from previous targets were strong, even ones associated with low-reward or no-reward. At the same time, however, we showed that monetary reward could influence attention. People found a high-reward target faster than a low- or no-reward target in the training phase, even though they may not have explicit knowledge about the reward association. Our study adds to the growing literature on rewardbased attention by illustrating both its limitation and its power.

Acknowledgments The authors thank Anthony Asaad, Michaela DeBolt, Gracelynn Goh, Hyejin Lee, Simon Ozbec, Joshua Shupe, and Xinyi Wang for help with data collection, and Leo Chelazzi, Roger Remington, Jan Theeuwes, and Nick Turk-Browne for comments and suggestions.

\section{References}

Anderson, B. A. (2013). A value-driven mechanism of attentional selection. Journal of Vision, 13(3). doi:10.1167/13.3.7

Anderson, B. A. (2015). Value-driven attentional priority is context specific. Psychonomic Bulletin \& Review, 22(3), 750-756. doi:10.3758/ s13423-014-0724-0

Anderson, B. A., Laurent, P. A., \& Yantis, S. (2011a). Value-driven attentional capture. Proceedings of the national academy of sciences of the United States of America, 108(25), 10367-10371. doi:10. 1073/pnas. 1104047108

Anderson, B. A., Laurent, P. A., \& Yantis, S. (2011b). Learned value magnifies salience-based attentional capture. PLoS ONE, 6(11), e27926. doi:10.1371/journal.pone.0027926 
Anderson, B. A., Laurent, P. A., \& Yantis, S. (2013). Reward predictions bias attentional selection. Frontiers in Human Neuroscience, 7, 262. doi:10.3389/fnhum.2013.00262

Anderson, B. A., Laurent, P. A., \& Yantis, S. (2014). Value-driven attentional priority signals in human basal ganglia and visual cortex. Brain Research, 1587, 88-96. doi:10.1016/j.brainres.2014.08.062

Anderson, B. A., \& Yantis, S. (2013). Persistence of value-driven attentional capture. Journal of Experimental Psychology. Human Perception and Performance, 39(1), 6-9. doi:10.1037/a0030860

Awh, E., Belopolsky, A. V., \& Theeuwes, J. (2012). Top-down versus bottom-up attentional control: Afailed theoretical dichotomy. Trends in Cognitive Sciences, 16(8), 437-443. doi:10.1016/j.tics.2012.06.010

Brainard, D. H. (1997). The Psychophysics Toolbox. Spatial Vision, 10(4), 433-436.

Bucker, B., Belopolsky, A. V., \& Theeuwes, J. (2015). Distractors that signal reward attract the eyes. Visual Cognition, 23(1-2), 1-24. doi: 10.1080/13506285.2014.980483

Chelazzi, L., Perlato, A., Santandrea, E., \& Libera, C. D. (2013). Rewards teach visual selective attention. Vision Research, 85, 58-72. doi:10. 1016/j.visres.2012.12.005

Della Libera, C., \& Chelazzi, L. (2006). Visual selective attention and the effects of monetary rewards. Psychological Science, 17(3), 222227. doi:10.1111/j.1467-9280.2006.01689.x

Della Libera, C., \& Chelazzi, L. (2009). Learning to attend and to ignore is a matter of gains and losses. Psychological Science, 20(6), 778784. doi:10.1111/j.1467-9280.2009.02360.x

Failing, M. F., \& Theeuwes, J. (2014). Exogenous visual orienting by reward. Journal of Vision, 14(5), 6. doi:10.1167/14.5.6

Folk, C. L., \& Remington, R. W. (2015). Unexpected abrupt onsets can override a top-down set for color. Journal of Experimental Psychology. Human Perception and Performance, 41(4), 11531165. doi:10.1037/xhp0000084

Folk, C. L., Remington, R. W., \& Johnston, J. C. (1992). Involuntary covert orienting is contingent on attentional control settings. Journal of Experimental Psychology. Human Perception and Performance, 18(4), 1030-1044.

Godwin, H. J., Menneer, T., Riggs, C. A., Cave, K. R., \& Donnelly, N. (2015). Perceptual failures in the selection and identification of lowprevalence targets in relative prevalence visual search. Attention, Perception \& Psychophysics, 77(1), 150-159. doi:10.3758/ s13414-014-0762-8

Gong, M., \& Li, S. (2014). Learned reward association improves visual working memory. Journal of Experimental Psychology. Human Perception and Performance, 40(2), 841-856. doi:10.1037/ a0035131

Gottlieb, J., Hayhoe, M., Hikosaka, O., \& Rangel, A. (2014). Attention, reward, and information seeking. The Journal of Neuroscience: The Official Journal of the Society for Neuroscience, 34(46), 1549715504. doi:10.1523/JNEUROSCI.3270-14.2014

Hickey, C., Chelazzi, L., \& Theeuwes, J. (2014). Reward-priming of location in visual search. PLoS ONE, 9(7), e103372. doi:10.1371/ journal.pone. 0103372

Horstmann, G., \& Ansorge, U. (2006). Attentional shifts to rare singletons. Visual Cognition, 14(3), 295-325. doi:10.1080/ 13506280500236492

Hout, M. C., Walenchok, S. C., Goldinger, S. D., \& Wolfe, J. M. (2015). Failures of perception in the low-prevalence effect: Evidence from active and passive visual search. Journal of Experimental Psychology. Human Perception and Performance. doi:10.1037/ xhp0000053

Jiao, J., Du, F., He, X., \& Zhang, K. (2015). Social comparison modulates reward-driven attentional capture. Psychonomic Bulletin \& Review, 22(5), 1278-1284. doi:10.3758/s13423-015-0812-9

Kristjánsson, A., \& Campana, G. (2010). Where perception meets memory: A review of repetition priming in visual search tasks. Attention, Perception \& Psychophysics, 72(1), 5-18. doi:10.3758/APP.72.1.5
Kristjánsson, Á., Sigurjónsdóttir, Ó., \& Driver, J. (2010). Fortune and reversals of fortune in visual search: Reward contingencies for pop-out targets affect search efficiency and target repetition effects. Attention, Perception, \& Psychophysics, 72(5), 1229-1236. doi:10. 3758/APP.72.5.1229

Kyllingsbæk, S., Schneider, W. X., \& Bundesen, C. (2001). Automatic attraction of attention to former targets in visual displays of letters. Perception \& Psychophysics, 63(1), 85-98. doi:10.3758/ BF03200505

Le Pelley, M. E., Pearson, D., Griffiths, O., \& Beesley, T. (2015). When goals conflict with values: Counterproductive attentional and oculomotor capture by reward-related stimuli. Journal of Experimental Psychology: General, 144(1), 158-171. doi:10.1037/xge0000037

Leber, A. B., \& Egeth, H. E. (2006). Attention on autopilot: Past experience and attentional set. Visual Cognition, 14(4-8), 565-583.

Lee, J., \& Shomstein, S. (2014). Reward-based transfer from bottom-up to top-down search tasks. Psychological Science, 25(2), 466-475. doi:10.1177/0956797613509284

Maljkovic, V., \& Nakayama, K. (1994). Priming of pop-out: I. Role of features. Memory \& Cognition, 22(6), 657-672.

Menneer, T., Cave, K. R., \& Donnelly, N. (2009). The cost of search for multiple targets: Effects of practice and target similarity. Journal of Experimental Psychology. Applied, 15(2), 125-139. doi:10.1037/ a0015331

Mine, C., \& Saiki, J. (2015). Task-irrelevant stimulus-reward association induces value-driven attentional capture. Attention, Perception, \& Psychophysics, 77(6), 1896-1907. doi:10.3758/s13414-015-0894-5

Miranda, A. T., \& Palmer, E. M. (2013). Intrinsic motivation and attentional capture from gamelike features in a visual search task. Behavior Research Methods, 46(1), 159-172. doi:10.3758/s13428013-0357-7

Monsell, S. (2003). Task switching. Trends in Cognitive Sciences, 7(3), 134-140.

Neo, G., \& Chua, F. K. (2006). Capturing focused attention. Perception \& Psychophysics, 68(8), 1286-1296. doi:10.3758/BF03193728

Pelli, D. G. (1997). The VideoToolbox software for visual psychophysics: Transforming numbers into movies. Spatial Vision, 10(4), 437-442.

Perkins, D. N., \& Salomon, G. (1992). Transfer of learning. In International encyclopedia of education (2nd ed.). Oxford: Pergamon Press.

Pool, E., Brosch, T., Delplanque, S., \& Sander, D. (2014). Where is the chocolate? Rapid spatial orienting toward stimuli associated with primary rewards. Cognition, 130(3), 348-359. doi:10.1016/j. cognition.2013.12.002

Qi, S., Zeng, Q., Ding, C., \& Li, H. (2013). Neural correlates of rewarddriven attentional capture in visual search. Brain Research, 1532, 32-43. doi:10.1016/j.brainres.2013.07.044

Roper, Z. J. J., Vecera, S. P., \& Vaidya, J. G. (2014). Value-driven attentional capture in adolescence. Psychological Science. doi:10.1177/ 0956797614545654

Sali, A. W., Anderson, B. A., \& Yantis, S. (2014). The role of reward prediction in the control of attention. Journal of Experimental Psychology. Human Perception and Performance, 40(4), 16541664. doi:10.1037/a0037267

Schmidt, L. J., Belopolsky, A. V., \& Theeuwes, J. (2015a). Attentional capture by signals of threat. Cognition and Emotion, 29(4), 687694. doi:10.1080/02699931.2014.924484

Schmidt, L. J., Belopolsky, A. V., \& Theeuwes, J. (2015b). Potential threat attracts attention and interferes with voluntary saccades. Emotion, 15(3), 329-338. doi:10.1037/emo0000041

Seitz, A., \& Watanabe, T. (2005). A unified model for perceptual learning. Trends in Cognitive Sciences, 9(7), 329-334. doi:10.1016/j.tics. 2005.05.010

Shen, Y. J., \& Chun, M. M. (2011). Increases in rewards promote flexible behavior. Attention, Perception \& Psychophysics, 73(3), 938-952. doi:10.3758/s13414-010-0065-7 
Shiffrin, R. M., \& Schneider, W. (1977). Controlled and automatic human information processing: II. Perceptual learning, automatic attending and a general theory. Psychological Review, 84(2), 127-190. doi:10. 1037/0033-295X.84.2.127

Shuler, M. G., \& Bear, M. F. (2006). Reward timing in the primary visual cortex. Science (New York, N.Y.), 311(5767), 1606-1609. doi:10. 1126/science. 1123513

Stankevich, B. A., \& Geng, J. J. (2014). Reward associations and spatial probabilities produce additive effects on attentional selection. Attention, Perception \& Psychophysics. doi:10.3758/s13414-014-0720-5

Swallow, K. M., \& Jiang, Y. V. (2013). Attentional load and attentional boost: A review of data and theory. Frontiers in Psychology, 4, 274. doi:10.3389/fpsyg.2013.00274

Theeuwes, J., \& Belopolsky, A. V. (2012). Reward grabs the eye: Oculomotor capture by rewarding stimuli. Vision Research, 74, 80-85. doi:10.1016/j.visres.2012.07.024
Vickery, T. J., Chun, M. M., \& Lee, D. (2011). Ubiquity and specificity of reinforcement signals throughout the human brain. Neuron, 72(1), 166-177. doi:10.1016/j.neuron.2011.08.011

Vickery, T. J., King, L.-W., \& Jiang, Y. (2005). Setting up the target template in visual search. Journal of Vision, 5(1), 81-92. doi:10. $1167 / 5.1 .8$

Wang, L., Yu, H., \& Zhou, X. (2013). Interaction between value and perceptual salience in value-driven attentional capture. Journal of Vision, 13(3), 5. doi:10.1167/13.3.5

Wentura, D., Müller, P., \& Rothermund, K. (2013). Attentional capture by evaluative stimuli: Gain- and loss-connoting colors boost the additional-singleton effect. Psychonomic Bulletin \& Review, 21(3), 701-707. doi:10.3758/s13423-013-0531-z

Wolfe, J. M. (2012). Saved by a log: How do humans perform hybrid visual and memory search? Psychological Science, 23(7), 698-703. doi: $10.1177 / 0956797612443968$ 